\title{
Modeling filter material with an orthotropic structure based on woven mesh
}

\author{
Aliaksandr Ilyushchanka ${ }^{1,2}$, Iryna Charniak $^{2}$, Vyacheslav Kaptsevich ${ }^{3}$, Valeria Korneeva $^{3}$, Ruslan Kusin $^{3}$, Igar Zakrevsky ${ }^{3}$, \\ and Pavel Chuhayeu ${ }^{3}$ \\ ${ }^{I}$ State Research and Production Powder Metallurgy Association, 220005 Minsk, Platonov str.41, Republic of Belarus \\ ${ }^{2}$ State Scientific Institution "O.V. Roman Powder Metallurgy Institute”, 220005 Minsk, Platonov str.41, Republic of Belarus \\ ${ }^{3}$ Belarusian State Agrarian Technical University, Minsk, Republic of Belarus
}

\begin{abstract}
A description is given of a filter material with an orthotropic structure based on woven mesh; its advantages are considered, and design options for filter elements from this material are given that are of practical interest for cleaning various liquids, including transmission ones. A model is proposed and the results of calculations of the structural and hydrodynamic properties of a filter material with an orthotropic structure based on woven mesh are presented.
\end{abstract}

\section{Introduction}

Mesh filter materials (MFMs) based on metal wire mesh are widely used for cleaning various liquids and gases. MFMs have a number of advantages over other filter materials (FMs), due to the combination of high strength and permeability; stability of the porous structure, which excludes the migration of FM particles into the cleaned medium; high heat resistance; and the ability to multiple regeneration [1, 2]. To produce MFM with high performance, it is reasonable to use woven metal mesh (WMM) with square cells. A significant disadvantage of such MFM is the low dirt capacity due to the fact that the method of surface filtering is implemented instead of a deep one in their practical use. On this account, filter element (FE) from MFM must often be replaced or regenerated [3]. At the same time, the regeneration process is difficult. It is impossible to completely remove all impurity particles stuck in the cells. Their failure to pass when using FE after regeneration is not guaranteed and the cells themselves are subject to deformation. However, using simple design and technological solutions, it is possible to to create new FMs applying MFMs, operating in the deep filtering mode. It is possible with their help to realize multi-stage purification of liquids and gases. Such MFMs can be almost completely cleaned of trapped particles of contaminants during the regeneration process. Such FMs can be produced by simple packing (laying by stack) of mesh with square cells, when the warp and weft wires are located one above the other, respectively $[4,5]$. The image of the structure of such a material is shown in Figure 1. In this case the structure can be called orthotropic, since it is obvious that in two mutually perpendicular directions, both the pores (namely, the fineness of cleaning) and the throughput (permeability coefficient) are significantly different.
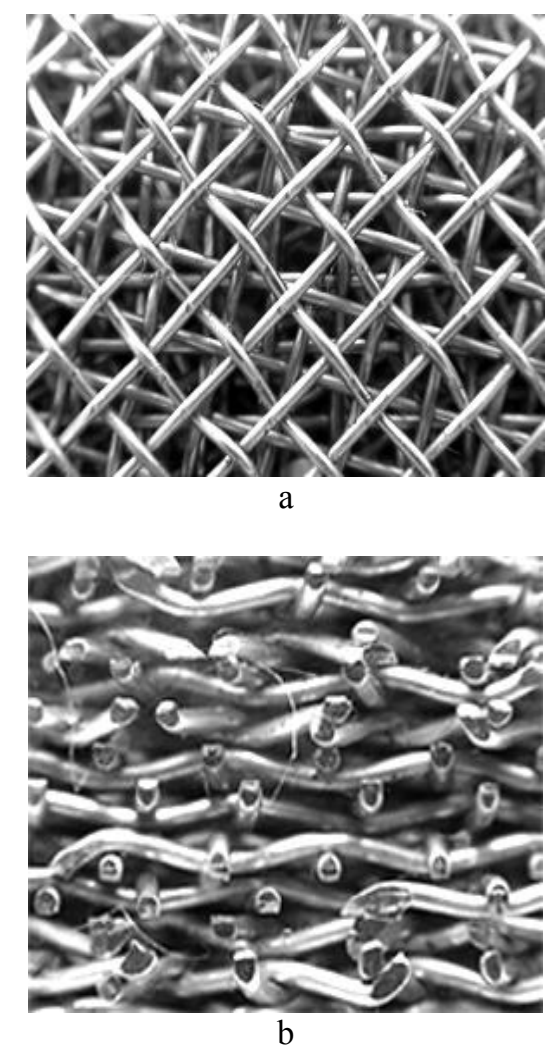

Fig. 1. MFM structure: $a-a$ view in the direction perpendicular to the plane of laying the meshes; $b-a$ view in a direction parallel to the plane of laying the meshes (The data source in its own development)

Two basic FE designs with an orthotropic structure based on WMMs are shown in Figure 2 and they reveal how the filtering ability of the laying is realized, shown in Figure $1, \mathrm{~b}$.

\footnotetext{
Corresponding author: irinacharniak@tut.by
} 

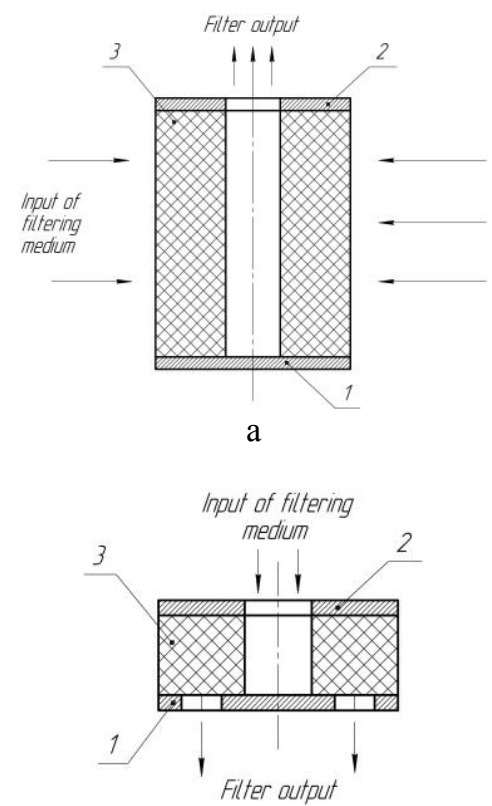

$\mathrm{b}$

Fig. 2. The operation scheme of a cylindrical (a) and flat (b) FEs with an orthotropic structure based on woven metal meshes: 1,2 - bottom and upper flanges,

3 - a packet of meshes (The data source in its own development).

Figure 3 shows images of a filter as an example that has been successfully used for cleaning herbicides. Similar filters are of interest for pre-treatment of transmission fluid in steady-state conditions.

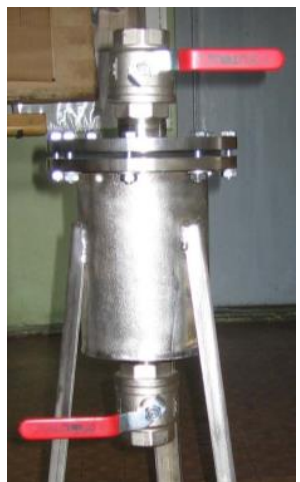

a

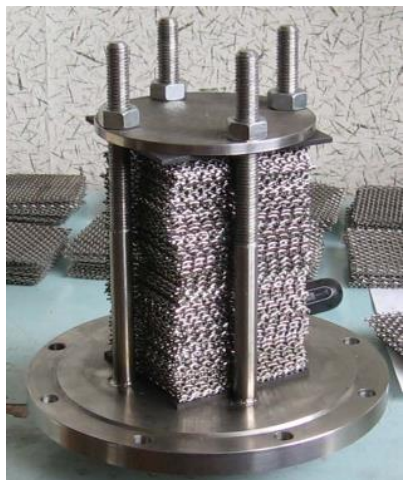

b
Fig. 3. Filter for cleaning herbicides: a - readyassembled filter; $b$ - filter element (The data source in its own development).

It is obvious that, in comparison with traditional filtering of the medium to be cleaned through the cells of the woven meshes, the use of the orthotropy of their structure makes it possible to reduce the fineness of cleaning and facilitate the regeneration process: when developing a filter element, the contour of the elementary filter cell becomes open, and the contaminants on the filter material are retained only by adhesion forces.
In accordance with the foregoing, a prediction of the properties of such materials at the design stage is of great interest.

The objective of this paper is to develop a model for calculating the properties of filter materials with an orthotropic structure based on woven metal meshes.

\section{Experimental results and discussion}

When developing the model, we will use an approach based on the building unit cells.

Figure 4 shows a model representation of a fragment of the material under consideration based on WMMs with square cells.

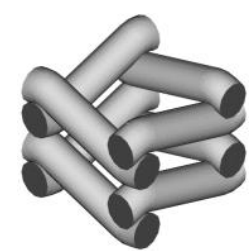

a

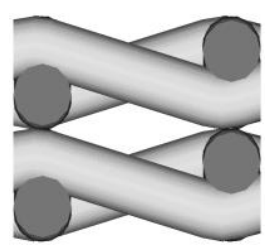

b

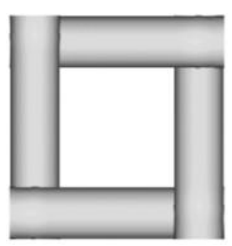

c
Fig. 4. A fragment of the design of a bulk mesh material: $\mathrm{a}$ - isometry; $\mathrm{b}$ - side view; $\mathrm{c}$ - top view (The data source in its own development).

Then the FM unit cell with an orthotropic structure based on woven metal meshes can be represented as a rectangular parallelepiped (Figure 5).

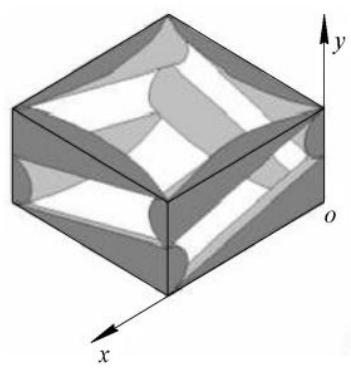

a

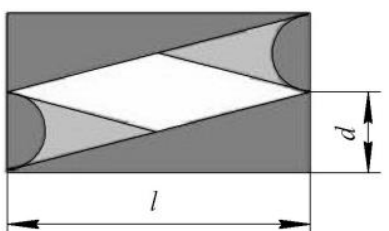

$\mathrm{b}$

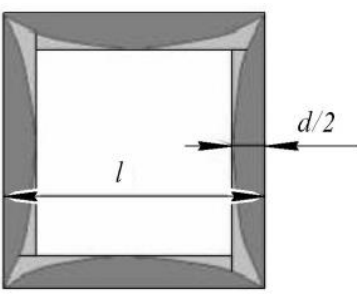

c

Fig. 5. Unit cell model: $\mathrm{a}$ - isometry; $\mathrm{b}$ - side view; $\mathrm{c}-$ top view (The data source in its own development). 
The dimensions of the presented cell are characterized by the diameter of the wire $d$ and the step of weaving the wire in the grid $l$.

According to reference [6], the structural properties of FM include porosity, specific surface area, and pore sizes.

Porosity $P$ is determined by the ratio of the pore volume $V_{\text {п }}$ to the total volume of the porous body $V[6]$.

For the considered unit cell $V=2 d l^{2}$ and $V_{\mathrm{n}}=2 d l^{2}-\frac{\pi}{2} d^{2} l$.

Then

$$
\mathrm{P}=1-\frac{\pi d}{4 l} .
$$

The specific surface $S_{\text {spec. }}$ is equal to the ratio of the surface area of the frame of the unit cell $S_{\text {surf }}$ to its volume $V$.

As follows from Figure $5 S_{\text {surf }}=2 \pi d l$. Then

$$
S_{\text {уд }}=\frac{\pi}{l} .
$$

The pore sizes are determined by the maximum circle diameters inscribed in the cross sections of the faces of the unit cell and will be characterized by values $d_{\Pi x}$ in the direction of the $O X$ axis and $d_{\Pi y}$ in the direction of the $O Y$ axis

$$
d_{\mathrm{rx}}=d, \quad d_{\mathrm{r} y}=l-d .
$$

The hydrodynamic properties of the material are determined by the permeability coefficients at various filtration modes. The calculation of the viscosity coefficient $k_{\mu}$ and inertial permeability coefficient $k_{\rho}$ is carried out by analogy with the calculation of the hydrodynamic properties of the cellular material considered in reference [7]. For this purpose, we use the well-known binominal equation of filtration that describes the fluid flow in a porous medium:

$$
\frac{\Delta p}{H_{0}}=\frac{\mu}{k_{\mu}} v+\frac{\rho}{k_{\rho}} v^{2},
$$

where $\Delta p$ - pressure drop on a layer of porous material; $H_{0}$ - filtering layer thickness; $\rho$ - fluid density; $\mu$-dynamic viscosity of fluid; $v$ - filtration rate.

Let us consider the fluid flow in a unit cell along the $O X$ axis. In this case, we distinguish two regions of fluid passage through the unit cell (Figure 6), namely: I - the region lying at the input and output of the fluid flow from the unit cell, and II - the region located in the center of the unit cell.

To determine the values of $k_{\mu x}$ and $k_{\rho x}$, it is necessary to calculate a number of auxiliary quantities.

The unit cell height $h$ and its cross-sectional area $S_{0}$ are equal to $h=2 d$, and $S_{0 x}=2 d l$, respectively.

Cross-sectional areas of region I $-S_{1 x}$ and region II $-S_{2 x}$ :

$$
S_{1 x}=d(l-\pi d / 4), S_{2 x}=d(2 l-\pi d / 4) .
$$
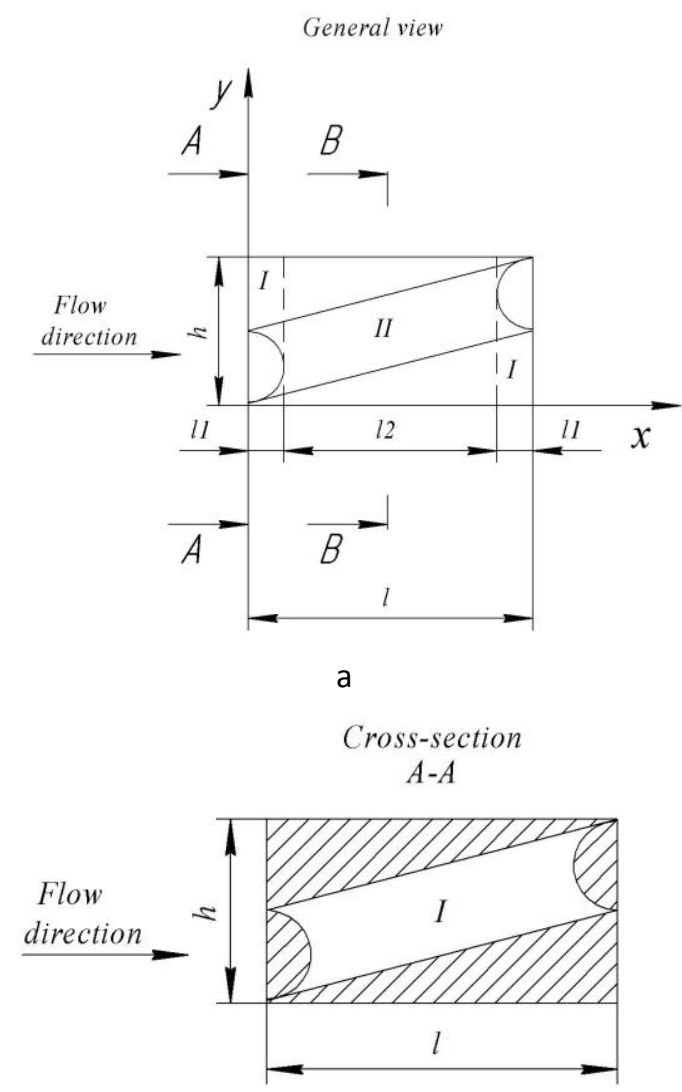

b

Cross-section

$B-B$

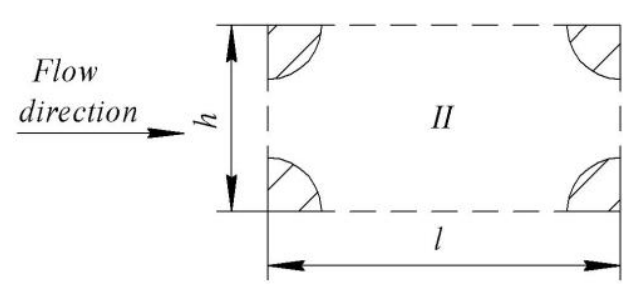

c

Fig. 6. Scheme of fluid flow through the unit cell of the MFM model along the $O X$ axis: a - cell division into regions; $\mathrm{b}$ - cross-section of region $\mathrm{I}-\mathrm{A}-\mathrm{A}$; $\mathrm{c}$ - crosssection of region $\mathrm{I}-\mathrm{B}-\mathrm{B}$ (The data source in its own development).

Cross-section perimeters of region I $-N_{1 x}$ and region II $-N_{2 x}$ :

$$
N_{1 x}=\pi d+2 \sqrt{d^{2}+l^{2}}, N_{2 x}=\pi d .
$$

The lengths of region $\mathrm{I}-l_{1 x}$ and region II $-l_{2 x}$ are defined as

$$
l_{1 x}=d, \quad l_{2 x}=l-d .
$$

From the continuity of the flow $v S_{0}=v_{1 x} S_{1 x}=v_{2 x} S_{2 x}$, we find the fluid velocities $v_{1 x}$, and $v_{2 x}$, respectively in regions I and II 


$$
v_{1 x}=v \frac{2 l}{l-\pi d / 4}, \quad v_{2 x}=v \frac{2 l}{2 l-\pi d / 4} .
$$

The hydraulic radii of the cross-sections of region $\mathrm{I}-R_{1 x}$ and region II $-R_{2 x}$ are equal to

$$
\begin{gathered}
R_{1 x}=\frac{S_{1 x}}{N_{1 x}}=\frac{d(l-\pi d / 4)}{\pi d+2 \sqrt{d^{2}+l^{2}}}, \\
R_{2 x}=\frac{S_{2 x}}{N_{2 x}}=\frac{2 l-\pi d / 4}{\pi} .
\end{gathered}
$$

Reynolds criteria $\mathrm{Re}_{1 x}, \mathrm{Re}_{2 x}$ for flow in regions I and II are equal to

$$
\begin{gathered}
\operatorname{Re}_{1 x}=\frac{v_{1 x} \rho R_{1 x}}{\eta}=\frac{v \rho}{\eta} \cdot \frac{2 l d}{\pi d+2 \sqrt{d^{2}+l^{2}}}, \\
\operatorname{Re}_{2 x}=\frac{v_{2 x} \rho R_{2 x}}{\eta}=\frac{v \rho}{\eta} \cdot \frac{2 l}{\pi} .
\end{gathered}
$$

The pressure loss on viscous friction within the unit cell is determined by the Darcy-Weisbach formula:

$$
\Delta p_{\mu x}=\frac{8 l_{1 x} \rho v_{1 x}^{2}}{\operatorname{Re}_{1 x} R_{1 x}}+\frac{8 l_{2 x} \rho v_{2 x}^{2}}{\operatorname{Re}_{2 x} R_{2 x}} .
$$

Then, using expressions (8-11), we obtain

$$
\Delta p_{\mu x}=16 v \mu l\left(\frac{\left(\pi d+2 \sqrt{d^{2}+l^{2}}\right)^{2}}{d(l-\pi d / 4)^{3}}+\frac{\pi^{2}(l-d)}{(2 t-\pi d / 4)^{3}}\right) \text {. }
$$

Thetefore, the pressure loss on viscous friction in a porous body of thickness $H$ increases by $H / h$ times, expression (12) takes the following form:

$$
\frac{\Delta p_{\mu x}}{H}=8 v \mu \frac{l}{d}\left(\frac{\left(\pi d+2 \sqrt{d^{2}+l^{2}}\right)^{2}}{d(l-\pi d / 4)^{3}}+\frac{\pi^{2}(l-d)}{(2 l-\pi d / 4)^{3}}\right) .
$$

Inertial pressure loss during the passage of fluid through the unit cell can be estimated by the Weisbach formula

$$
\Delta p_{\rho x}=\frac{w_{1} \rho\left(v-v_{1 x}\right)^{2}}{2}+\frac{w_{2} \rho\left(v_{1 x}-v_{2 x}\right)^{2}}{2},
$$

where $w_{1}, w_{2}$ - coefficients of pressure drop losses. In this case, we assume that $w_{1}=w_{2}=w$.

Then, inserting exprisson (8) into (14), we obtain

$$
\Delta p_{\rho x}=\frac{w \rho v^{2}}{2}\left(1-\frac{2 l}{l-\pi d / 4}\right)^{2}+2 w \rho v^{2}\left(\frac{l}{l-\pi d / 4}-\frac{l}{2 l-\pi d / 4}\right)^{2} .
$$

Similarly with the pressure losses on viscous friction, the inertial losses in a porous body of thickness $H$ will also increase by $N / h$ times. Therefore, expression (15) will take the following form:

$$
\frac{\Delta p_{\rho x}}{H}=\frac{w \rho v^{2}}{4 d}\left(1-\frac{2 t}{l-\pi d / 4}\right)^{2}+\frac{w \rho v^{2}}{d}\left(\frac{l}{l-\pi d / 4}-\frac{l}{2 l-\pi d / 4}\right)^{2}
$$

Then the total pressure loss in the porous body will be

$$
\frac{\Delta p}{H}=\frac{\Delta p_{\mu}}{H}+\frac{\Delta p_{\rho}}{H}=\frac{\mu}{k_{\mu}} v+\frac{\rho}{k_{\rho}} v^{2} .
$$

We insert (13) and (16) into expression (17) and define $k_{\mu x}$ and $k_{\rho x}$ in the form

$$
\begin{gathered}
\frac{1}{k_{\mu x}}=8 \frac{l}{d}\left(\frac{\left(\pi d+2 \sqrt{d^{2}+l^{2}}\right)^{2}}{d(l-\pi d / 4)^{3}}+\frac{\pi^{2}(l-d)}{(2 l-\pi d / 4)^{3}}\right), \\
\frac{1}{k_{\rho x}}=\frac{w}{4 d}\left(1-\frac{2 l}{l-\pi d / 4}\right)^{2}+\frac{w}{d}\left(\frac{l}{l-\pi d / 4}-\frac{l}{2 l-\pi d / 4}\right)^{2} .
\end{gathered}
$$

In a similar way, the hydrodynamic properties of the material can be calculated for the case when the fluid flow is directed along the $O Y$ axis.

\section{Conclusion}

Promising designs of filter materials are described with an orthotropic structure based on woven metal mesh capable to undergo multiple regenerations with complete purification from contaminants without changing operational characteristics. Examples of their successful operation are given. The model is proposed for describing the structure and calculating the properties of filter materials with an orthotropic structure based on woven metal mesh.

\section{References}

1. Y. Sinelnikov, Porous mesh materials, Moscow, Metallurgy (1983).

2. R. Timirkeev, V. Sapozhnikov, Industrial purity and fine filtration of working fluids for aircraft vehicles, Moscow, Mechanical Engineering (1986).

3. I. Bray, Y. Kudinov, I. Belyavsky, Filters for diesel fuel fine purification, Moscow, Mashgiz (1963).

4. Patent of the Republic of Belarus No. 4811, IPC 7 B 01D 29/44, Slotted Filter, P. Vityaz, R. Kusin, I. Valkovich, V. Kaptsevich, V. Krugley; application No. 19980607; declared 06/29/98. Publ. 07/22/2002.

5. Patent of the Republic of Belarus No. 949. U. B 01D 29/00, Composite Filter, V. Kaptsevich, A. Krutov, V. Korneeva, G. Azarau, R. Kusin, G. Bokan, I. Lykov, A. Kusin. Application u 20020395. Publ. 09/30/2003. Official Bulletin No. 3, 2003.

6. P. Vityaz, V. Kaptsevich, R. Kusin, Filter materials: properties, areas of applications, manufacturing technology, Minsk, Research Institute of PM with PP, (1999).

7. A. Leonov, M. Dechko, V. Sheleg, Porous permeable materials: theory of product design and technology, Minsk, Tonpik (2003). 